\title{
Pipelines and Systems for Threshold Avoiding Quantification of LC-MS/MS data
}

\author{
Alejandro Sánchez Brotons \\ University of Groningen \\ Jonatan Eriksson \\ Lund University \\ Marcel Kwiatkowski \\ University of Innsbruck \\ Justina Wolters \\ University Medical Center Groningen https://orcid.org/0000-0003-0066-3720 \\ Ido Kema \\ University Medical Center Groningen \\ Andrei Barcaru \\ University Medical Center Groningen \\ Folkert Kuipers \\ University Medical Center Groningen \\ Stephan Bakker \\ University Medical Center Groningen \\ Rainer Bischoff \\ University of Groningen https://orcid.org/0000-0001-9849-0121 \\ Frank Suits \\ IBM Research Australia \\ Peter Horvatovich ( $\square$ p.l.horvatovich@rug.nl) \\ University of Groningen https://orcid.org/0000-0003-2218-1140
}

\section{Article}

Keywords: LC-MS/MS pre-processing, proteomics, label-free, isotope quantification

Posted Date: May 5th, 2021

DOI: https://doi.org/10.21203/rs.3.rs-422254/v2

License: (c) (1) This work is licensed under a Creative Commons Attribution 4.0 International License. Read Full License 
Version of Record: A version of this preprint was published at Analytical Chemistry on August 6th, 2021. See the published version at https://doi.org/10.1021/acs.analchem.1c01892. 


\title{
Pipelines and Systems for Threshold Avoiding Quantification of LC-MS/MS data
}

\author{
Alejandro Sánchez Brotons ${ }^{1}$, Jonatan O. Eriksson ${ }^{2}$, Marcel Kwiatkowski ${ }^{1,3}$, Justina C. \\ Wolters $^{4}$, Ido P. Kema ${ }^{5}$, Andrei Barcaru ${ }^{1}$, Folkert Kuipers ${ }^{4,5}$, Stephan J.L. Bakker ${ }^{6}$, \\ Rainer Bischoff ${ }^{1}$, Frank Suits ${ }^{7}$, Péter Horvatovich ${ }^{1, *}$ \\ ${ }^{1}$ University of Groningen, Department of Analytical Biochemistry, Groningen Research Institute of \\ Pharmacy, Antonius Deusinglaan 1, 9713 AV, Groningen, The Netherlands. \\ ${ }^{2}$ Lund University, Department of Biomedical Engineering, Lund. \\ ${ }^{3}$ Functional Proteo-Metabolomics, Department of Biochemistry, University of Innsbruck, Innsbruck, Austria. \\ ${ }^{4}$ Department of Pediatrics, University Medical Center Groningen, University of Groningen, Groningen, The \\ Netherlands. \\ ${ }^{5}$ Department of Laboratory Medicine, University Medical Center Groningen, University of Groningen, 9700 \\ RB Groningen, The Netherlands. \\ ${ }^{6}$ Department of Internal Medicine, Division of Nephrology, University Medical Center Groningen, \\ University of Groningen, Groningen, The Netherlands. \\ ${ }^{7}$ IBM Research - Australia. 60 City Rd., Southbank VIC 3006, Australia. \\ *Corresponding author
}

\section{ORCIDs}

ASB : $0000-0003-4578-8957$

JCW : 0000-0003-0066-3720

FK : 0000-0003-2518-737X

FS : 0000-0001-6427-9299

\section{Correspondence}

Peter Horvatovich

E-mail: p.l.horvatovich@rug.nl

\section{Keywords}

LC-MS/MS pre-processing, proteomics, label-free, isotope quantification
JE : 0000-0003-3015-2630
MK: 0000-0002-5804-6031
IK : 0000-0003-1166-6169
AB : $0000-0002-8549-8244$
SJLB : 0000-0003-3356-6791
RB : 0000-0001-9849-0121 


\begin{abstract}
The accurate processing of complex LC-MS/MS data from biological samples is a major challenge for metabolomics, proteomics and related approaches. Here we present the Pipelines and Systems for Threshold Avoiding Quantification (PASTAQ) LC-MS/MS pre-processing toolset, which allows highly accurate quantification of data-dependent acquisition (DDA) LC-MS/MS datasets. PASTAQ performs compound quantification using single-stage (MS1) data and implements novel algorithms for high-performance and accurate quantification, retention time alignment, feature detection, and linking annotations from multiple identification engines. PASTAQ offers straightforward parametrization and automatic generation of quality control plots for data and pre-processing assessment. This design results in smaller variance when analyzing replicates of proteomes mixed with known ratios, and allows the detection of peptides over a larger dynamic concentration range compared to widely used proteomics preprocessing tools. The performance of the pipeline is also demonstrated in a biological human serum dataset for the identification of gender related proteins.
\end{abstract}

\title{
Introduction
}

Liquid chromatography coupled to tandem mass spectrometry (LC-MS/MS) is a powerful analytical technique for the quantitative profiling of proteins, peptides and metabolites in complex biological samples. In the last decade, advances in instrumentation such as faster acquisition speed, improved sensitivity and increased dynamic range have made LC-MS/MS the method of choice for routine analyses in clinical and life science applications [1,2], as well as a widely used tool for biomarker discovery, quantitative protein and metabolite profiling and drug screening [3-6].

LC-MS/MS data are complex and require the use of sophisticated data pre-processing pipelines that allow to extract quantitative and identification information of compounds. For this purpose, numerous tools and pipelines exist, both commercial and freely-available ones, such as MaxQuant [7], OpenMS [8] and XCMS [9]. Some of these tools are used mainly for proteomics, while others are more flexible in their usage, with support for metabolomics or lipidomics analyses. In general, tools developed for label-free data-dependent acquisition (DDA) proteomics applications rely on the quantification of isotope clusters (features) which have been identified using MS/MS spectra to match the quantitative information of the same peptide across multiple samples. One limitation of this approach is that for DDA analyses, only a fraction of 
the most abundant compounds is selected for fragmentation. Furthermore, identifications might not be possible for all compounds due to the inherent stochasticity of the selection of precursor ions for fragmentation in the MS/MS sampling process, and thus, compounds present in the sample in a low concentration (or compounds that ionize poorly) will be less likely to be selected for fragmentation.

Chimeric spectra can also occur with typical DDA fragmentation windows of $0.4-2.0 \mathrm{~m} / \mathrm{z}$ [10], making the correct identification of peptides challenging. Singlestage (MS1) spectra in DDA LC-MS/MS data reflect a stable quantitative profile of compounds that can be detected by the mass spectrometer, and are not subjected to a stochastic selection procedure such as DDA precursor selection for compound fragmentation. We argue that MS1 data offer the most stable information to perform comprehensive and accurate processing of DDA LC-MS/MS data sets. This approach relies only on the $\mathrm{m} / \mathrm{z}$ and retention time information of the compounds' features and depends on accurate correction of any shifts existing in these separation domains.

Here, we present the Pipelines and Systems for Threshold Avoiding Quantification (PASTAQ), a set of tools and algorithms that can be used to pre-process and quantify compounds present in LC-MS/MS data, regardless of the existence of MS/MS spectra. PASTAQ is built on the algorithmic and workflow design of the Threshold Avoiding Proteomics Pipeline (TAPP) [11], which is focused on accurately processing single-stage LC-MS data at the isotope level. PASTAQ performs the quantification of LC-MS/MS compounds exclusively with MS1 information, and MS/MS based identification is used for annotation of matched MS1 features. PASTAQ includes an improved version of the Warp2D retention time alignment algorithm and allows assessment of alignment accuracy by evaluating the similarity of the chromatograms after retention time alignment. PASTAQ offers a comprehensive set of tools, a pre-built DDA pipeline and an easy to use GUI that enables the quantification and exploration of LC-MS/MS data tailored for metabolomics and proteomics analyses. Furthermore, PASTAQ allows the use of any identification engine that supports exporting identifications in the mzIdentML format [12], including post-processing tools such as PeptideShaker [13], PeptideProphet [14], ProteinProphet [15] or Percolator [16] to adjust the false discovery rate (FDR).

To evaluate both the quantitative and computational performance of this pipeline we used two different datasets. The first one consists of a proteome mixture of HeLa, yeast, and Escherichia coli (E. coli) at three different ratios: (A) 10:5:1 (B) 5:10:1 (C) 1:5:10. The second dataset is composed of a HeLa matrix with an artificial concatemer protein [17] spiked in at increasing ratios to cover three orders of magnitude of compound 
concentration and concomitant measured ion intensities. The first and second datasets are hereinafter referred to as the HYE dataset and the QconCAT dataset, respectively. Both datasets were acquired with a nano LC system coupled to an Orbitrap QExactive Plus mass spectrometer. Additionally, we demonstrate the use of PASTAQ with publicly available serum LC-MS/MS datasets [18] to show its performance with a biologically relevant dataset.

\section{Methods}

\section{Preparation of complex proteome samples}

\section{HYE dataset}

To generate complex proteome samples with known composition a tryptic HeLa protein digest (Pierce ${ }^{\mathrm{TM}}$ HeLa Protein Digest Standard, Thermo Fisher Scientific, Dreieich, Germany), a tryptic yeast protein digest (Mass Spec-Compatible Yeast Extract, Promega, Walldorf, Germany) and an Escherichia coli (E. coli) tryptic protein digest (Waters, Manchester, UK) were used. For differential proteomics, three proteome mixtures (A, B, C) were prepared, composed of the HeLa proteome, yeast proteome, and E. coli proteome. A) $20 \mu \mathrm{g}$ of HeLa digest (dissolved in $0.1 \% \mathrm{FA}$ ) was combined with $10 \mu \mathrm{g}$ yeast digest (dissolved in $0.1 \%$ FA) and $2 \mu \mathrm{g}$ E. coli digest (dissolved in $0.1 \%$ FA). B) $10 \mu \mathrm{g}$ of HeLa digest (dissolved in $0.1 \%$ FA) was combined with $20 \mu \mathrm{g}$ yeast digest (dissolved in $0.1 \%$ FA) and $2 \mu \mathrm{g}$ E. coli digest (dissolved in $0.1 \%$ FA). C) $2 \mu \mathrm{g}$ of HeLa digest (dissolved in $0.1 \%$ FA) was combined with $10 \mu \mathrm{g}$ yeast digest (dissolved in $0.1 \%$ FA) and $20 \mu \mathrm{g}$ E. coli digest (dissolved in $0.1 \%$ FA). The sample mixtures A, B and C had a final concentration of $2 \mu \mathrm{g} \mathrm{L}^{-1}$ (dissolved in $0.1 \% \mathrm{FA}$ ).

\section{QconCAT dataset}

The complex proteome samples with the spiked isotopically-labelled standards covering the three orders of magnitude in dynamic range were prepared with MS compatible Human Protein Extract (V694A, whole-cell protein extract prepared from human H562 cells, Promega) was digested with an in-gel digestion protocol as described previously [17]. Digests were resuspended in $0.1 \%$ FA at a final concentration of $1 \mu \mathrm{g} \mu \mathrm{L}^{-1}$ and mixed 1:1 with isotopically-labeled standard peptides. The isotopically-labeled peptides were digested from the three concatamers targeting a specific set of mitochondrial proteins as described previously [17]. The standard peptides were added at 
increasing $\log _{10} \mathrm{ng} / \mathrm{\mu g}$ amounts from -3.0 up to 1.0.

\section{LC-MS/MS analysis}

For LC-MS/MS analysis, $1 \mu \mathrm{L}$ were injected on a nano-ultra pressure liquid chromatography system (Dionex UltiMate 3000 RSLCnano pro flow, Thermo Scientific, Bremen, Germany) coupled via nano electrospray-ionization (ESI) to a quadrupole orbitrap mass spectrometer equipped with a nano-electrospray ion source (Orbitrap Q Exactive Plus, Thermo Scientific, Bremen, Germany). Chromatographic separation of the peptides was performed using a nano-LC column (Acclaim PepMapC100 C18, $75 \mu \mathrm{m}$ x $50 \mathrm{~cm}, 2 \mu \mathrm{m}, 100 \AA$, Dionex, buffer A: $0.1 \% \mathrm{v} / \mathrm{v}$ formic acid (FA), dissolved in $\mathrm{H}_{2} \mathrm{O}$, buffer b: $0.1 \% \mathrm{v} / \mathrm{v}$ FA, dissolved in acetonitrile (ACN)). The peptides were loaded onto a trap column ( $\mu$ Precolumn cartridge, Acclaim PepMap100 C18, $5 \mu \mathrm{m}, 100 \AA$,

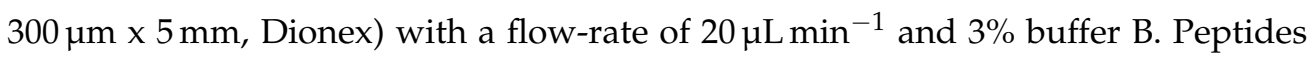
were separated on the nano-LC column with a flow-rate of $300 \mathrm{~nL} \mathrm{~min}^{-1}$ using a linear gradient from 3-30\% buffer B in $85 \mathrm{~min}$, followed by $30-50 \%$ buffer B in $5 \mathrm{~min}$. The mass spectrometer was operated in positive ion mode and data-dependent acquisition mode (DDA) using a top-10 method. MS spectra were acquired at a resolution of 70,000 at m/z 200 over a scan range of 350 to $1300 \mathrm{~m} / \mathrm{z}$ with a AGC target of $10^{6}$ ions and a maximum injection time of $50 \mathrm{~ms}$. Peptide fragmentation was performed with higher energy collision dissociation (HCD) using a normalized collision energy (NCE) of 28. The intensity threshold for ions selection was set at $2.0 \cdot 10^{4}$ with a charge exclusion of $\leq 1$ and $\geq 7$. The MS/MS spectra were acquired at a resolution of 17,500 at $200 \mathrm{~m} / \mathrm{z}$, a AGC target of $10^{5}$ ions and a maximum injection time of $50 \mathrm{~ms}$ and the quadrupole isolation window set to $1.6 \mathrm{~m} / \mathrm{z}$. The same instrument was used for the QconCAT dataset with the following alterations compared to the HYE dataset: a total of $3 \mu \mathrm{L}$ was injected and the mass spectrometer was operated with a top-15 method, with a charge exclusion of $\leq 1$ and $\geq 5$. The raw data are available via ProteomeXchange with identifier PXD024584.

\section{Pre-processing and parametrization details}

\section{Format conversion}

The raw Orbitrap data files were processed directly in MaxQuant (Version 1.6.10.43), but PASTAQ requires files in mzXML [19] or mzML [20] format and SearchGUI [21] (Version 3.3.17) only works properly with centroided mgf [22] files. File conversion was 
performed with ProteoWizard's msConvert [23] (Version 3.0.18342-01b48c0f0), with a binary encoding precision of 64 bits. The mzXML conversion was performed without additional processing. For the mgf conversion, centroiding was performed using the vendor's peak picking algorithm included in msConvert.

\section{Peptide and protein identification}

For protein identification UP000005640 (Homo sapiens, updated on August 21, 2019), UP000002311 (Baker's yeast, updated on July 25, 2019), and UP000000625 (Escherichia coli, strain K12, updated on August 21, 2019) Swissprot [24] protein sequences were used. The canonical sequence of each proteome was downloaded in FASTA format from the UniProt Knowledgebase (UniProtKB) website on October 31, 2019.

Prior to identification, the FASTA files of the three proteomes were concatenated. This FASTA file was used directly in MaxQuant [7] and MSFragger [25] (Version 3.1.1). When using SearchGUI [21], this FASTA file was modified with the addition of reverse decoy sequences using the built in tools.

Fixed modification of cysteine by carbamidomethylation $(\mathrm{C})$ and the variable modification of methionine through oxidation to the sulfoxide $(\mathrm{M})$ were used for all datasets. The QconCAT dataset was searched with the additional variable modifications of ${ }^{13} \mathrm{C}+6$ on lysine $(\mathrm{K})$ and arginine $(\mathrm{R})$ due to the presence of stable isotopes in these amino acids for this artificial protein.

SearchGUI was used to perform peptide and protein identification with the aforementioned FASTA file and PSM settings. The precursor mass tolerance was set to $5 \mathrm{ppm}$ and the fragment ion mass tolerance to $0.02 \mathrm{Da}$. The search engines selected for identification were (1) X!Tandem [26], (2) MS Amanda [27] and (3) MS-GF+ [28]. The SearchGUI results were unified into a consensus mzIdentML [12] identification file for each sample using PeptideShakerCLI (Version 1.16.42) [13] and less than 1\% FDR at PSM, peptide and protein levels.

\section{HYE analysis}

We performed an exhaustive analysis of quantified isotopes, features, peptides and protein groups obtained with PASTAQ and MaxQuant. When PASTAQ quantification was used, we used SearchGUI/PeptideShaker for identifications.

For both PASTAQ and MaxQuant, we assigned the corresponding "Human", "Yeast" or "E. coli" proteome to each of the identified clusters. In case a cluster contained 
a consensus identification sequence that could belong to more than one proteome, said cluster was not considered for the analysis. Clusters without any linked identification information were assigned an "Unknown" proteome. For consistency in the comparisons, only clusters within the retention time range between $1500 \mathrm{~s}$ and $5700 \mathrm{~s}$, since data outside of this region contain mostly contaminants or undigested proteins. In the case of MaxQuant, the data are clustered only at the peptide ("peptides.txt") and protein levels ("proteinGroups.txt"). Thus, to assess feature level quantification the results from the "evidence.txt" file were grouped by the combination of peptide sequences and charge states.

The following statistics are calculated overall (all samples) and per group (A, B and C): (1) mean, (2) median, (3) standard deviation, (4) CV, (5) median of $\log _{10}$ transformed data, (6) standard deviation of $\log _{10}$ transformed data, (7) percentage of zero values and (8) number of zero values. Afterwards, the difference in median $\log _{10}$ data between sample groups A and C, A and B, and B and C was calculated.

To explore the influence of intensity on the distribution of $\mathrm{CVs}$, we generated 2D density plots of median $\log _{10}$ intensity vs CV\% for each sample group (Fig. S1).

Finally, the accuracy of the quantification was assessed by comparing the $\log _{10}$ ratio of the different groups as a factor of the intensity with the known expected values for each ratio: $\log _{10}(\mathrm{~A} / \mathrm{C})$ vs $\log _{10}(\mathrm{C}), \log _{10}(\mathrm{~A} / \mathrm{B})$ vs $\log _{10}(\mathrm{~B})$, and $\log _{10}(\mathrm{~B} / \mathrm{C})$ vs $\log _{10}(\mathrm{C})$. For each of these groups, scatterplots of median $\log _{10}$ values were generated alongside a corresponding density plot (Fig. S2). Similarly, the overall distribution of median $\log _{10}$ values in each proteome was also explored in the form of violinplots (Fig. S3).

\section{QconCAT analysis}

For the analysis of the QconCAT dataset, the goal was to study the effect of the dynamic range of compounds on the quantitative performance. We decided to focus on feature level quantification. The applied QconCATs are artificial proteins created from concatenated peptides selected for the intended human and mouse targets. After digestion, the peptides only differ from the endogenous peptides by the presence of the isotope-label that each peptides carries on the lysines or arginines

To ensure that the features being compared are the same across pipelines, we selected only those features in which the peptide sequence and charge states were the same, and for which retention times and $\mathrm{m} / \mathrm{z}$ are within $\pm 50 \mathrm{~s}$ and $\pm 0.05 \mathrm{~m} / \mathrm{z}$, respectively.

The wide range of three orders of magnitude of spiked-in relative amounts means 
that we could not assume that detected QconCAT compounds will follow a linear distribution throughout the entire range. Furthermore, to perform linear fitting between the $\log _{10} \mathrm{ng} / \mathrm{\mu g}$ and $\log _{10}$ intensity values on any given feature cluster, we started by considering all the points in the cluster for the model fit and assessing the resulting $R^{2}$. If $R^{2}<0.98$, we iteratively removed low intensity values until the constraint is satisfied. To avoid spurious matches, we only considered features in which the aforementioned linear fit contain values in at least three spiked-in levels. If the fitting is successful, the CV, and SSE are calculated for all points in the fitted linear range. Only features that were successfully fitted in both PASTAQ and MaxQuant were considered for further analysis. An example of the fitted models for different compounds can be found on Fig. S4. Additionally, we obtained the extracted ion chromatograms (XIC) of the monoisotopic peaks for all selected features after retention time alignment (Fig. S5) with an $\mathrm{m} / \mathrm{z}$ window of \pm 0.01 .

\section{Theory}

Pre-processing of DDA data comprises detecting isotopic peaks or peptide features, aligning the retention time of all chromatograms to a common reference, linking isotopic peaks and features with identification information, matching peaks/features across chromatograms and generating quantitative tables amenable for statistical analysis.

The parametrization of PASTAQ is very intuitive to anyone familiar with LC-MS instrumentation settings and theory since it is based on the well-understood notions of peak widths and compound separation in chromatography and mass spectrometry. PASTAQ calculates the theoretical width of peaks in the retention time and $\mathrm{m} / \mathrm{z}$ dimensions by considering the physics of the ion separation and detection process in different MS instruments [11] as well as chromatographic separation theory [29]. For example, peak width modeling in mass spectra is based on the ion separation equation of the mass analyzers, while chromatographic peak modeling is based on the assumption of a constant peak width, as predicted by the linear solvent strength theory for reverse phase linear gradient elution [29].

Another important feature of PASTAQ is the automatic generation of quality control plots, which allow to assess the overall dataset similarity between samples, distribution of peak widths, as well as retention time and mass shifts. These quality control plots can be used to evaluate the quality of the pre-processed data and identify issues with 
the acquired LC-MS/MS data or with the given parametrization (Fig. S6). The default parameters were established to accurately process data acquired with the most common acquisition settings, and the user is only required to set three key parameters: the type of mass spectrometer used, the resolution at the reference $\mathrm{m} / \mathrm{z}$, and the average full width at half maximum (FWHM) of chromatographic peaks. Many other parameters in the pipeline, such as the selection of regions of interest for peak detection, the level of smoothing, and the radius for feature/peak matching across chromatograms, are automatically derived from those parameters.

One of the goals of PASTAQ is to provide full data traceability from beginning to end of the analysis. This allows for post-hoc analyses and data exploration. For example, by tracing back all processing steps and intermediate results that comprise a quantified peptide, we can find each fragment ion spectrum associated with the isotopic peaks in all available files and display relevant information of retention time and mass shifts in all datasets. The spectra associated with a matched isotope or matched peptide feature can also be extracted from the raw data.

The core of PASTAQ is built as a C++ library, with bindings in the Python programming language. It can be used in all major operating systems (i.e. Windows, Mac, and Linux), including high-performance computing clusters. This allows PASTAQ to be easily integrated into existing workflows and LC-MS/MS analysis pipelines. Additionally, the Python bindings enable PASTAQ to be extended to suit the needs of different datasets and allow quick iteration and prototyping of new ideas, such as the generation of consensus spectra from matched and/or identified MS/MS spectra. All code is available under a permissive open source license (MIT) and is publicly available at https://pastaq.horvatovichlab.com.

This combination of features makes PASTAQ suitable for beginners and advanced users alike. Running the basic pipeline is simple, but more complex analyses can be performed using the Python bindings to derive further insights from the data.

Many of the steps necessary for LC-MS/MS data pre-processing can be computationally demanding. For instance, when considering a large number of samples, millions of isotopic peaks need to be quantified, aligned, and matched with suitable candidates throughout the entire dataset. Moreover, selecting the proper pre-processing parameters can be challenging due to the complexity of LC-MS/MS data, which often needs to be analyzed multiple times to test different identification or quantification parameters. PASTAQ circumvents this by separating the identification and quantification steps, and the main pre-processing algorithms try to take advantage of multi-core processing when available. 
The datasets presented here were analyzed on a workstation with an Intel(R) Core(TM) i7-8700K CPU running at $3.7 \mathrm{GHz}$ and $64 \mathrm{~GB}$ of RAM memory running Linux 5.9.3. The data were stored in four Western Digital WD Blue 6TB hard drives in a RAID10 configuration under the BTRFS file system.

A brief description of the main pre-processing modules of the DDA pipeline is provided below, and a more detailed explanation can be found in the Supplementary Information. A simplified diagram of how the different modules are connected in PASTAQ's DDA pipeline can be seen in Fig. 1.

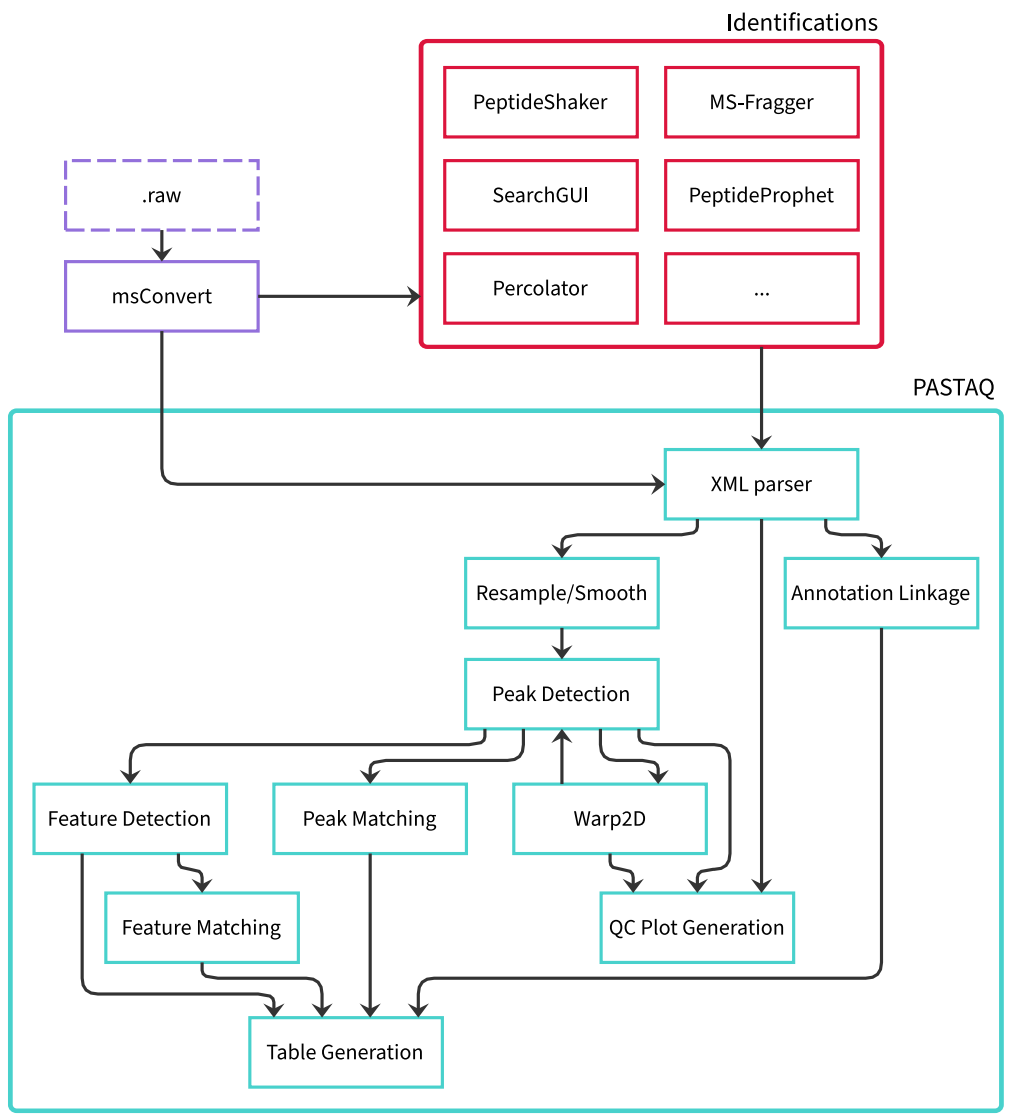

Figure 1: Diagram of the interactions between the main modules in PASTAQ's DDA pipeline. A more detailed diagram can be found in the Supplementary Information (Fig. S7). Note that identifications are not required for peak or feature detection by PASTAQ but necessary to link features to identified peptides and ultimately to proteins. 


\section{Results}

\section{Peak detection and feature detection}

PASTAQ detects isotopic peaks by considering their three-dimensional nature in LCMS/MS data. While mass spectra are smoothed in Orbitrap high-resolution mass spectrometers on the acquisition electronic board, which reduces the noise in the $\mathrm{m} / \mathrm{z}$ dimension, noise is unaffected in the chromatographic dimension. Time of Flight and quadrupole-based mass spectrometers tend to have a higher level of noise in both separation dimensions. The width of peaks in the $\mathrm{m} / \mathrm{z}$ dimension is highly dependent on the instrument resolution at a given $\mathrm{m} / \mathrm{z}$, as well as the type of mass spectrometer used. For accurate quantification, PASTAQ performs a simultaneous 2D Gaussian kernel smoothing and resampling of the spectra that maps the acquired raw data into a regular grid, keeping the number of sampling points per isotope peak constant throughout the entire $\mathrm{m} / \mathrm{z}$ range independently of their width. This results in consistently sized peaks across the entire $\mathrm{m} / \mathrm{z}$ range as well as reduced memory usage.

The smoothed grid is searched for local maxima, and a novel, fast method for non-iterative 3D Gaussian fitting is used to minimize the error of the Gaussian model applied to the raw data using the location of peak maxima in the smoothed grid. This results in superior quantitative performance whilst being computationally efficient, which makes PASTAQ suitable for processing large datasets in a reasonable time. This process results in a list of modeled peaks for each chromatogram, describing their $\mathrm{m} / \mathrm{z}$ and retention time location as well as their height and width in the respective dimensions. In our work, PASTAQ detected an average of 550,393 isotopic peaks for the chromatograms of the HYE dataset and 398, 521 for the QconCAT dataset.

For this first stage of the pipeline, it took an average of 17 seconds to parse 6GB profile mzXML files for MS1 spectra and 33 seconds for MS2. Peak detection was performed in around 10 seconds for resampling and 4 seconds for peak detection, 2D Gaussian fitting and quantification per file. The total mzXML parsing time for the 30 samples in the HYE dataset, including saving the detected raw spectra to disk in binary format, was 32 minutes. The peak detection procedure finished in less than 40 minutes.

It is often desirable to group peaks belonging to the same isotopic envelope into "features". To achieve this, PASTAQ uses the previously obtained peak lists to generate undirected graphs in which peaks are tentatively linked if they are within a close retention time range and their $\mathrm{m} / \mathrm{z}$ location difference corresponds to 1.0033 divided by the candidate charge state. The range of candidate charge states is set by the user, and 
should be typically between 1 and 8 . The tolerance for retention time and $\mathrm{m} / \mathrm{z}$ is set as a unit of peak width (sigma) in the respective dimensions. Features are formed by using these graphs to find the best matching isotopic patterns to the appropriate averagine model [30]. By using this approach, an average of 150,793 features were obtained for the HYE dataset and 116, 519 for the QconCAT dataset. This corresponds to respective averages of 3.42 and 3.65 isotopes per detected feature. Despite the inherent complexity of the task, feature detection was performed in around 4 seconds per file and less than 5 minutes for all the 30 samples in the HYE dataset.

\section{Retention time alignment}

Aligning the retention time of all chromatograms in a dataset is a crucial step to be able to match compounds between samples using a predominantly MS1 based approach. For this purpose, PASTAQ uses an improved version of the Warp2D algorithm [31]. This method allows the alignment of two chromatograms by maximizing the similarity function of their respective peak lists based on sum of overlapping 2D Gaussian peaks. An extra benefit of this approach is that the calculation of similarity values across all samples allows the identification of potential outliers. For example, in Fig. 2(a,b) the similarity matrix before and after alignment shows a remarkable improvement after Warp2D, where the replicates of the three different ratios of the HYE dataset are clearly differentiated.

Performing retention time alignment of two samples takes roughly 12 seconds with the default parameters. If a given sample is used as a reference, the 30 replicates of the HYE dataset can be aligned in less than 6 minutes. If no reference is given for retention time alignment, an exhaustive search is performed in order to select the best reference to maximize the average similarity for all samples. This optional step can become the most computationally expensive part of the pipeline for large datasets, as the time required will increase proportionally to the square of the number of samples in the dataset. In the case of large datasets, it is possible to skip this step or to select a subset of samples on which alignment between all pairs can be performed. For the HYE dataset, the exhaustive reference search took 86 minutes until completion.

\section{Peak matching and feature matching}

Matching peaks and features belonging to the same compounds across multiple samples is necessary for assessing relative differences in intensity. In PASTAQ, this task is greatly simplified thanks to the robust and accurate retention time alignment algorithm 

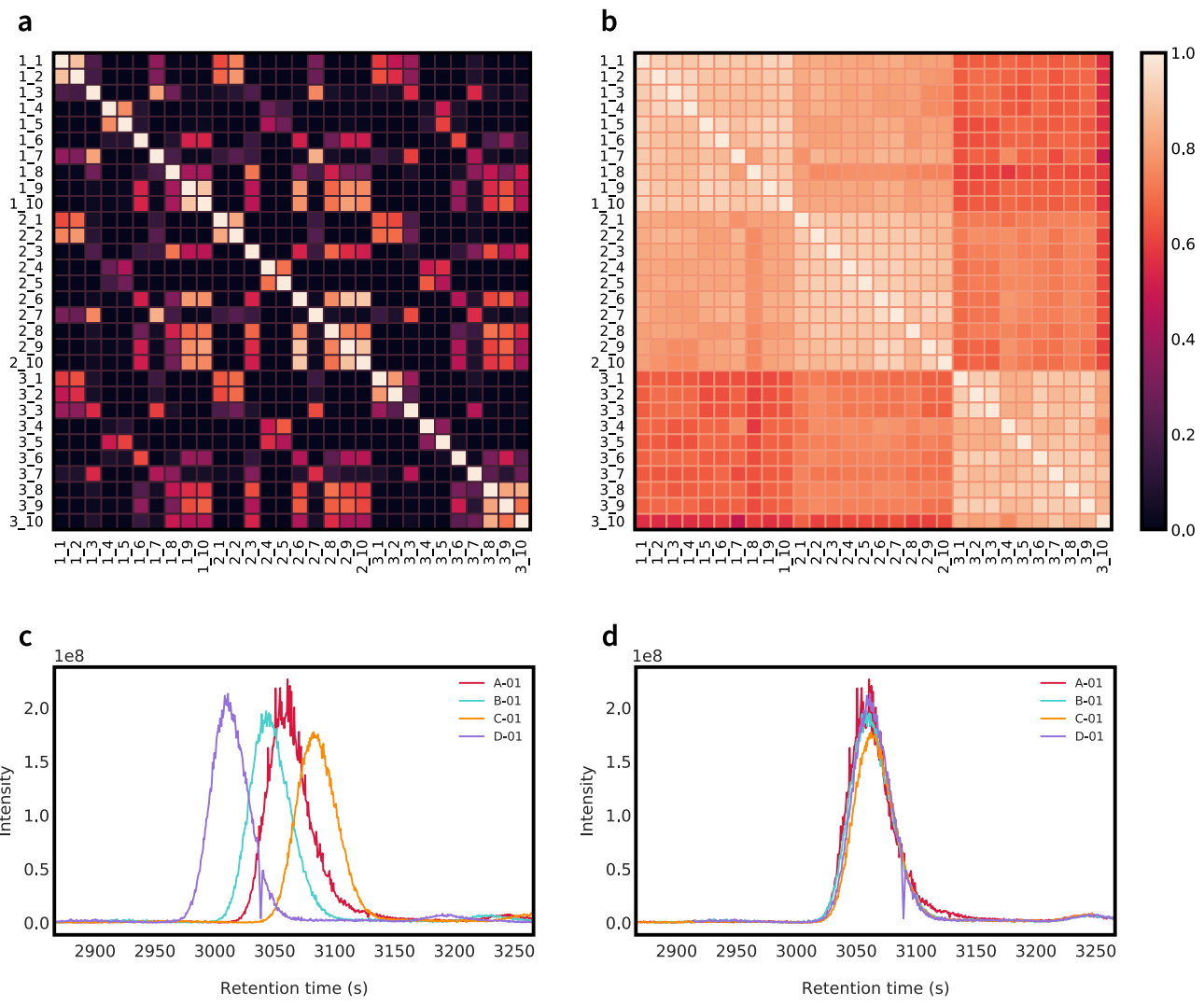

Figure 2: Effect of the retention time alignment on the similarity matrix of the HYE dataset (top) and the extracted ion chromatogram of an isotopic peak from the QconCAT dataset (bottom), before (a, c) and after (b, d) alignment.

used in the pipeline. The process consists of comparing the retention time and $\mathrm{m} / \mathrm{z}$ locations within a tolerance range dictated by a given number of sigmas in the retention time and $\mathrm{m} / \mathrm{z}$ dimensions. The matching is the same for peaks and features, but in the case of features, the monoisotopic $\mathrm{m} / \mathrm{z}$ is used and only features that share the same charge state (as determined by the feature matching procedure in PASTAQ) are considered. At the end of this process a list of clustered isotopic peaks and features is obtained.

To reduce the effect of noise on the data, an optional filtering step will keep the clusters in which at least a minimum percentage of samples from any of the groups 
under study contain detected values. Matching based exclusively on MS1 location allows consistent matching of all detected MS1 peaks or features independent of their identification. This approach avoids the common risk of identification-based methods, namely using two different types of matching for identified and unidentified peaks, where the latter is often based on identification transfer. The MS/MS-based annotation of MS1 peaks in individual samples allows to assess consistency of identification annotation in matched clusters and for the creation of a consensus identification for each cluster. With our matching algorithm, the matching of 639,543 isotopic peak clusters in the HYE dataset took 3 minutes and 30 seconds, and 65 seconds for 83, 295 feature clusters. Similarly, we obtained 828,801 isotopic peaks and 229, 307 feature clusters for the QconCAT dataset.

\section{MS1 based quantification improves accuracy, precision and number of quantified compounds}

Analysis of the chosen datasets mainly focuses on the quantitative performance of PASTAQ compared with the widely used MaxQuant software. The first quantitative metric assessed was the coefficient of variation $(\mathrm{CV})$ of each of the 10 replicates in each group for all measured peptides in the HYE dataset. The CV is calculated for features, peptides as well as protein groups. For MaxQuant, we used the "evidence.txt" file for assessment of features, the "peptides.txt" file for peptide level quantification, and the "proteinGroups.txt" file for protein group quantification. The combination of PASTAQ quantification with PeptideShaker consensus identifications resulted in lower CV between replicates, as indicated in Table 1. A more detailed overview of the analysis process can be found in the Supplementary Information. Furthermore, when comparing the distribution of the $\mathrm{CV}$ for all quantified features, peptides and proteins, PASTAQ has a smaller interquartile range. As shown in Fig. 3(a-c) and Table 1, this applies for features as well as protein groups, but the difference is smaller for the latter, likely due to the error inherent to the aggregation of peptides to proteins which relates to the so-called protein inference problem [32].

It is also important to mention that due to the stochasticity of fragmentation during DDA LC-MS/MS and the presence of multiple post-translational modifications of peptides, quantitative methods that rely on identification information might miss potentially relevant biological signals. An example of this issue is illustrated in Fig. S2, which shows that only a third of the features quantified by PASTAQ had linked identifications passing a 1\% FDR threshold. While some of these quantified features 


\begin{tabular}{ccccccccccc}
\hline & & \multicolumn{3}{c}{ Median CV (\%) } & \multicolumn{2}{c}{ Stdev CV (\%) } & \multicolumn{3}{c}{ IQR CV (\%) } \\
& & Human & Yeast & Ecoli & Human & Yeast & Ecoli & Human & Yeast & Ecoli \\
\hline \multirow{3}{*}{ PASTAQ } & features & 16.96 & 15.52 & 16.06 & 19.01 & 20.15 & 20.02 & 13.94 & 12.07 & 13.99 \\
& peptides & 18.25 & 15.67 & 17.36 & 24.65 & 25.96 & 24.56 & 17.79 & 15.08 & 17.37 \\
& proteins & 25.82 & 21.14 & 26.70 & 21.86 & 18.93 & 22.91 & 24.39 & 20.67 & 27.40 \\
\hline \multirow{3}{*}{ MaxQuant } & features & 23.06 & 20.55 & 21.25 & 20.80 & 21.44 & 22.36 & 20.07 & 18.08 & 20.21 \\
& peptides & 28.13 & 25.27 & 26.58 & 27.01 & 27.40 & 27.04 & 25.13 & 22.87 & 24.77 \\
& proteins & 34.18 & 26.43 & 31.70 & 25.14 & 24.09 & 24.91 & 31.10 & 26.94 & 30.33 \\
\hline
\end{tabular}

Table 1: Comparing the distribution of CV (\%) in the HYE dataset between PASTAQ (with SearchGUI + PeptideShaker identifications) and MaxQuant.

may be attributed to noise, it is evident that the distribution of these unidentified features mirrors closely that of the identified ones, albeit the former had lower intensity on average.

To assess how well the different pipelines perform to detect and quantify peptides spiked across three orders of magnitude we used the QconCAT dataset. The features reported in the "evidence.txt" file from MaxQuant were matched with the corresponding features from PASTAQ using their associated peptide sequence and charge state, excluding ambiguous identifications. For each of the matched features between PASTAQ and MaxQuant, a linear fitting was performed over the $\log _{10}$ transformed intensity vs the $\mathrm{ng}$ of spiked in protein over $\mu \mathrm{g}$ of total protein $(\mathrm{ng} / \mu \mathrm{g})$ to select the spiked-in levels that fall within the linear range (Fig. S4), as described in more detail in the Supplementary Information. This resulted in 118 features, for which the $\mathrm{CV}$, sum of squared errors (SSE) of the linear fit, and number of features present in replicates at each spiked-in level were calculated. While both pipelines performed similarly in groups with higher concatemer amount $[1.00-10.00 \mathrm{ng} / \mathrm{\mu g}]( \pm 5 \%$ difference in the number of quantified peptides), we observe an increase of $16.7 \%$ to $72.5 \%$ in the number of quantified peptides with PASTAQ compared to MaxQuant (using the match-between-runs option) when the spiked-in amount is below $1 \mathrm{ng} / \mu \mathrm{gg}$. All spiked-in features show similar or smaller CV and SSE in PASTAQ, as shown in Fig. 3(d-f) and Table 2.

\section{Threshold-avoiding quantification enables the detection of a larger number of quantified proteins in biological datasets}

To show the performance of PASTAQ for pre-processing biological LC-MS/MS data, we have used two label-free LC-MS/MS proteomics datasets of human serum acquired with a short gradient for blood based biomarker profiling [18]. First, we focused on 


\begin{tabular}{|c|c|c|c|c|}
\hline \multicolumn{2}{|c|}{ Spiked-in amount } & \multicolumn{2}{|c|}{ Num. peptides } & \multirow{2}{*}{ Difference (\%) } \\
\hline $\mathrm{ng} / \mathrm{\mu g}$ & $\log _{10}(\mathrm{ng} / \mu \mathrm{g})$ & PASTAQ & MaxQuant & \\
\hline 10.0000 & 1.0 & 374 & 381 & -1.85 \\
\hline 3.16230 & 0.5 & 371 & 370 & 0.27 \\
\hline 1.00000 & 0.0 & 354 & 336 & 5.36 \\
\hline 0.31623 & -0.5 & 301 & 258 & 16.67 \\
\hline 0.10000 & -1.0 & 181 & 139 & 30.22 \\
\hline 0.03162 & -1.5 & 69 & 40 & 72.50 \\
\hline 0.01000 & -2.0 & 8 & 8 & 0.00 \\
\hline 0.00316 & -2.5 & 4 & 0 & NA \\
\hline 0.00100 & -3.0 & 0 & 0 & NA \\
\hline
\end{tabular}

Table 2: Difference in the number of detected peptides between PASTAQ and MaxQuant for the different spiked-in levels in the QconCAT dataset.

b
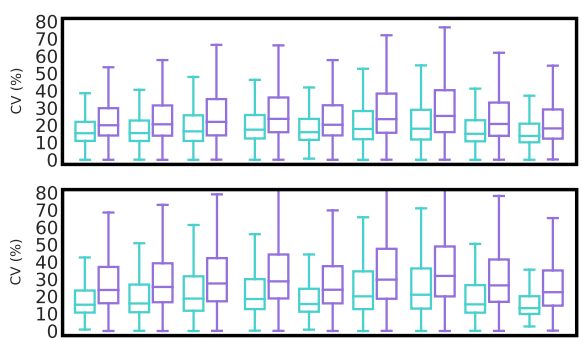

C

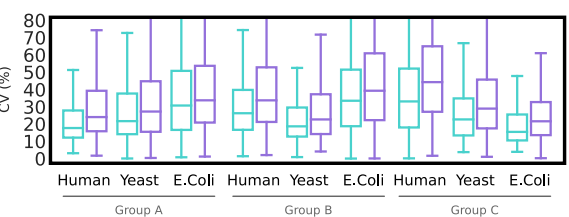

d

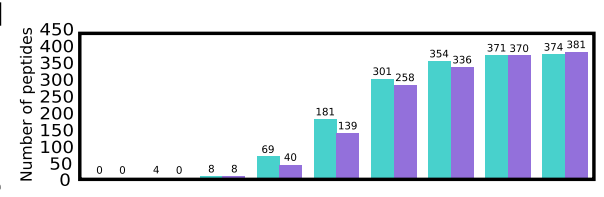

e

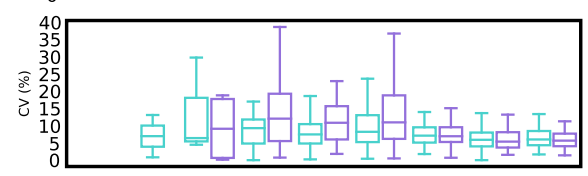

$f$

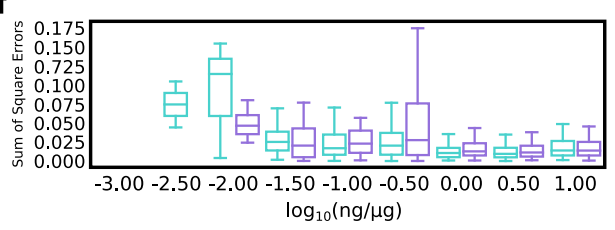

$=$ PASTAQ - - MaxQuant

Figure 3: $(\mathrm{a}-\mathrm{c})$ Comparative of the CV (\%) between PASTAQ quantification with PeptideShaker's consensus identifications, and MaxQuant for (a) features, (b) peptides and (c) proteins in the HYE dataset. (d-f) Evaluation of (d) number, (e) CV and ( $f$ ) sum of square errors of quantified peptide for each $\log _{10}$ spiked-in level in the QconCAT dataset. 
417

more than $60 \%$ of the replicates. The median $\mathrm{CV}$ of the quantified protein groups with PASTAQ was $14.6 \%$, which is between the values obtained with the original MaxQuant quantification (16.9\%) and after MaxLFQ [33] normalization (12.4\%). The standard deviation of the CV values is of $10.8 \%$ for PASTAQ, approximately 2 times lower than MaxQuant (23.9\%) or MaxLFQ (19.5\%), indicating the consistency of the quantification by PASTAQ when analyzing serum sample replicates (Fig. 4a). Additional normalization algorithms such as median fold-change normalization [34] might further reduce the CVs at the feature, peptide and/or protein levels.
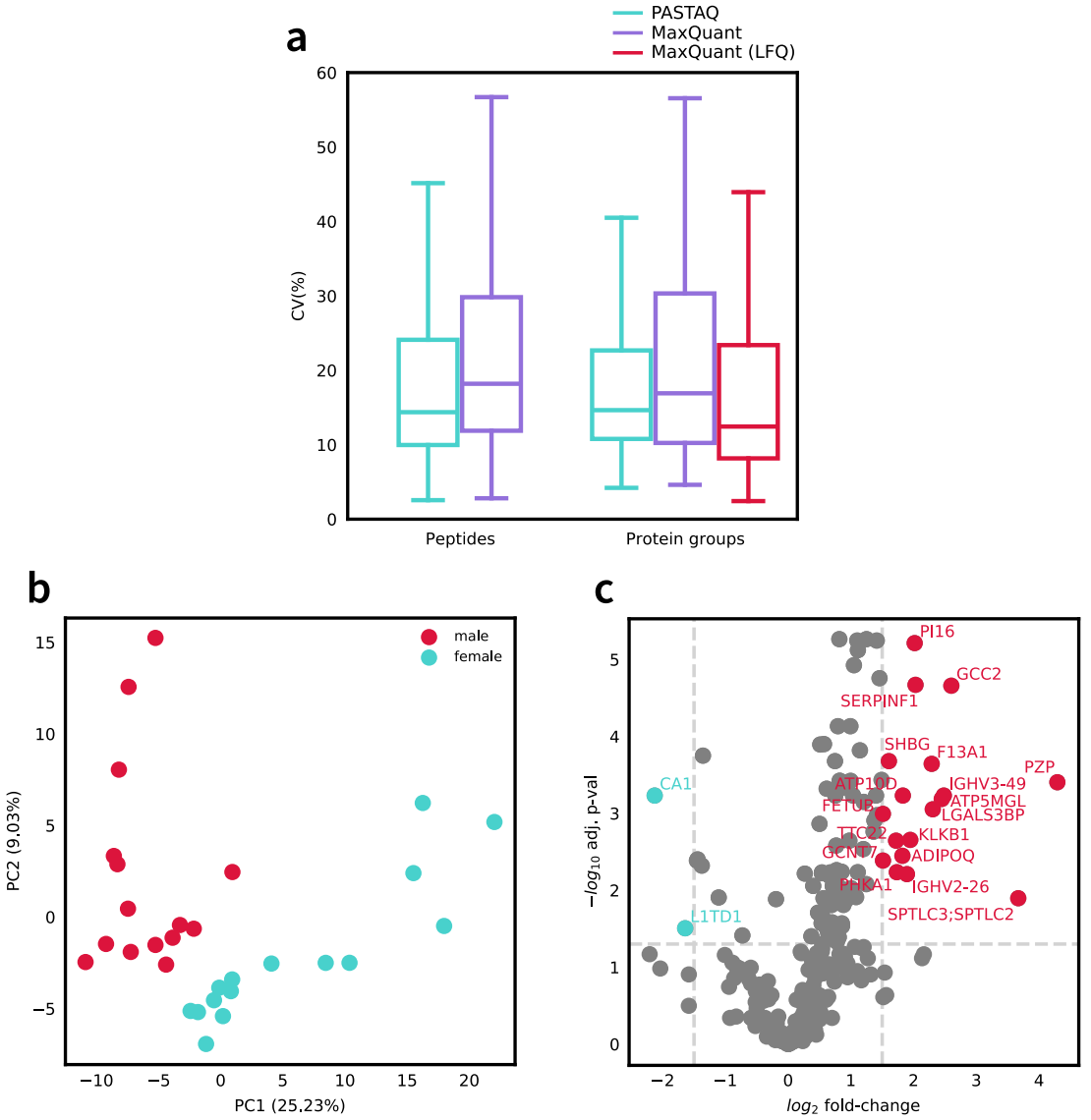

Figure 4: Evaluation of PASTAQ performance on human serum datasets: (a) Distribution of CVs on the peptide and protein group quantification levels for the fifteen technical replicates dataset. (b) Principal Component Analysis (PCA) of the male-female dataset, showing a clear separation between groups. (c) Volcano plot showing selected gender related proteins for the male-female dataset based on the adjusted p-values from Welch's t-test and the $\log _{2}$ fold-change. 
445

\section{a4a Discussion}

LC-MS/MS data are often used for biomarker discovery. To demonstrate the suitability of PASTAQ for this task, we processed the datasets from 5 male and 5 female subjects (in triplicate) from the aforementioned work of Geyer et al. The data processing parameterization and $60 \%$ filtering with PASTAQ were the same as in the previous example, yielding 291 protein groups. We performed a principal component analysis (PCA), in which a difference between the male and female groups can be clearly observed (Fig. 4b). A $\log _{2}$ transformation was applied to the data to account for its log-normal distribution. In addition, Welch's t-test was performed on each protein group and the resulting p-values were adjusted for multiple testing using the BenjaminiHochberg [35] method, resulting in 100 significant protein groups (adjusted $p<0.05$ ). The statistical results are visualized as a volcano plot in Fig. 4c. We focused on protein groups with a $\log _{2}$ fold-change of more than 1.5 , yielding several proteins already documented in the literature, including two of the reported significant proteins in the original work of Geyer et al.: Pregnancy zone protein (PZP) and sex hormon-binding globulin (SHBG). Furthermore, we found Fetuin-B (FETUB), Phospholipid-transporting ATPase VD (ATP10D) and Tetratricopeptide repeat protein 22 (TTC22) to be different between males and females. The level of FETUB is known to follow the menstrual cycle and correlates with women's fertility [36], while TTC22 and ATP10D are known to be highly expressed in female tissues based on the Human Protein Atlas [37-39].

In this work we show how the innovative design of the preprocessing and quantification methods of PASTAQ lead to the detection of large number of low intensity signals from LC-MS/MS data, whilst offering excellent reproducibility and accurate quantification for low abundant peptides and proteins. This is due to the combined use of smoothed and raw data for peak detection and quantification, the avoidance of early thresholding which enlarges the dynamic quantification range, the use of overlapping peak volumes in WARP2D retention time alignment to automatically and accurately align multiple chromatograms, as well as the exclusive MS1-based peak matching. Quality metrics such as exhaustive pairwise similarity matrices before and after alignment may be used to assess the accuracy of data processing. Linking pre-processing parameters to peak widths in the mass and retention time domains allows straightforward parameterization. Furthermore, the speed of the pre-processing algorithms used in PASTAQ allows rapid iteration of different parameters and/or identification engines 


\section{${ }_{484}$ Author contributions} remains a considerable challenge. exploration of LC-MS/MS datasets.

when analyzing large datasets, thus enabling an efficient parameter optimization in order to obtain higher quantification accuracy.

The stochasticity of fragmentation in DDA LC-MS/MS can lead to situations where no identification information is available for the detected features in the data. This has important consequences for experiments that emphasize the search for new biomarkers, since the information in the entire dataset can be used to select a list of candidates even when no identification is available. PASTAQ allows to collect all MS/MS spectra linked to matched peaks or features and allows manual interpretation of individual or consensus spectra in order to elucidate the compound to which they belong. This is particularly relevant for metabolomics and lipidomics datasets, where identification

In this manuscript we highlighted the use of PASTAQ for pre-processing DDA proteomics data, but the given tools are not limited to this domain. Data-independent acquisition (DIA) methods attempt to address the stochasticity issue by performing comprehensive fragmentation of the entire mass range using consecutive and large precursor selection windows. This method has proven to be more effective in extracting a large number of identifications from the data [40], but it has some disadvantages, including the increased complexity of data analysis due to heavily convoluted MS/MS spectra that require more elaborate approaches for data processing and quantification. We are actively working towards the implementation of novel DIA quantifications methods in PASTAQ as well as its usage with metabolomics and lipidomics datasets.

In summary, PASTAQ represents a step forward in LC-MS/MS data pre-processing. The straightforward set of tools in its suite allow the user to extract additional and more reliable biological insights from the data and identify potential sources of error, enabling faster analysis cycles, and providing the necessary tools for the in-depth

$\mathrm{PH}, \mathrm{FS}$ and RB designed and supervised the study; ASB, PH, FS and AB contributed to the design of computational algorithms, ASB designed and implemented PASTAQ; JOE performed independent testing of PASTAQ; MK and JCW performed sample preparation and acquisition of LC-MS/MS datasets; ASB, SJLB, IK, FK, MK, RB, and FS, discussed assessment and quality metrics and contributed to interpretation of the results; ASB and PH wrote the paper with contributions from all authors. 


\section{${ }_{491}$ Competing interests}

492 The authors declare that they have no competing financial interests.

\section{${ }_{493}$ Acknowledgements}

This research was part of the Netherlands X-omics Initiative and partially funded by NWO, project 184.034.019.

\section{References}

[1] Wiener, M. C., Sachs, J. R., Deyanova, E. G. \& Yates, N. A. Differential mass spectrometry: A label-free LC-MS method for finding significant differences in complex peptide and protein mixtures. Analytical Chemistry 76, 6085-6096 (2004).

[2] Levin, Y., Schwarz, E., Wang, L., Leweke, F. M. \& Bahn, S. Label-free LC-MS/MS quantitative proteomics for large-scale biomarker discovery in complex samples. Journal of Separation Science 30, 2198-2203 (2007). arXiv: 1011 .1669v3.

[3] McCormack, A. L. et al. Direct Analysis and Identification of Proteins in Mixtures by LC/MS/MS and Database Searching at the Low-Femtomole Level. Analytical Chemistry 69, 767-776 (1997).

[4] Maurer, H. H. Multi-analyte procedures for screening for and quantification of drugs in blood, plasma, or serum by liquid chromatography-single stage or tandem mass spectrometry (LC-MS or LC-MS/MS) relevant to clinical and forensic toxicology. Clinical Biochemistry 38, 310-318 (2005).

[5] Lee, M. S. \& Kerns, E. H. LC/MS applications in drug development. Mass Spectrometry Reviews 18, 187-279 (1999).

[6] Fernie, A. R., Trethewey, R. N., Krotzky, A. J. \& Willmitzer, L. Metabolite profiling: From diagnostics to systems biology. Nature Reviews Molecular Cell Biology 5, 763-769 (2004).

[7] Cox, J. \& Mann, M. MaxQuant enables high peptide identification rates, individualized p.p.b.-range mass accuracies and proteome-wide protein quantification. Nature Biotechnology 26, 1367-1372 (2008). nbt. 1511. 
[8] Weisser, H. et al. An automated pipeline for high-throughput label-free quantitative proteomics. Journal of Proteome Research 12, 1628-1644 (2013).

[9] Smith, C. A., Want, E. J., O'Maille, G., Abagyan, R. \& Siuzdak, G. XCMS: Processing mass spectrometry data for metabolite profiling using nonlinear peak alignment, matching, and identification. Analytical Chemistry 78, 779-787 (2006).

[10] Houel, S. et al. Quantifying the impact of chimera MS/MS spectra on peptide identification in large-scale proteomics studies. Journal of Proteome Research $\mathbf{9}$, 4152-4160 (2010).

[11] Suits, F., Hoekman, B., Rosenling, T., Bischoff, R. \& Horvatovich, P. Thresholdavoiding proteomics pipeline. Analytical Chemistry 83, 7786-7794 (2011). URL http://pubs.acs.org/doi/abs/10.1021/ac201332j.

[12] Mayer, G., Proteom-center, M., Bochum, R.-u. \& Eisenacher, M. mzIdentML: exchange format for peptides and proteins identified from mass spectra. Tech. Rep. (2011).

[13] Vaudel, M. et al. PeptideShaker enables reanalysis of MS-derived proteomics data sets: To the editor. Nature Biotechnology 33, 22-24 (2015).

[14] Keller, A. D., Nesvizhskii, A. I., Kolker, E. \& Aebersold, R. Empirical statistical model to estimate the accuracy of protein identifications made by MS/MS and database search. Proceedings 50th ASMS Conference on Mass Spectrometry and Allied Topics 74, 37-38 (2002).

[15] Nesvizhskii, A. I., Keller, A., Kolker, E. \& Aebersold, R. A statistical model for identifying proteins by tandem mass spectrometry. Analytical chemistry 75, 4646-58 (2003). URL http://www.ncbi.nlm.nih.gov/pubmed/14632076.

[16] Käll, L., Canterbury, J. D., Weston, J., Noble, W. S. \& MacCoss, M. J. Semisupervised learning for peptide identification from shotgun proteomics datasets. Nature Methods 4, 923-925 (2007).

[17] Wolters, J. C. et al. Translational Targeted Proteomics Profiling of Mitochondrial Energy Metabolic Pathways in Mouse and Human Samples. Journal of Proteome Research 15, 3204-3213 (2016).

[18] Geyer, P. E. et al. Plasma Proteome Profiling to Assess Human Health and Disease. Cell Systems 2, 185-195 (2016). URL http://dx.doi.org/10.1016/j.cels. 2016. 02.015. 
[19] Pedrioli, P. G. A. et al. A common open representation of mass spectrometry data and its application to proteomics research (2004). URL http://www . nature.com/ articles/nbt1031.

[20] Martens, L. et al. mzML-a Community Standard for Mass Spectrometry Data. Molecular \& Cellular Proteomics 10, R110.000133 (2011). URL http: //www.mcponline.org/lookup/doi/10.1074/mcp.R110.000133.

[21] Barsnes, H. \& Vaudel, M. SearchGUI: A Highly Adaptable Common Interface for Proteomics Search and de Novo Engines. Journal of Proteome Research 17, 2552-2555 (2018).

[22] Perkins, D. N., Pappin, D. J., Creasy, D. M. \& Cottrell, J. S. Probability-based protein identification by searching sequence databases using mass spectrometry data. Electrophoresis 20, 3551-3567 (1999).

[23] Chambers, M. C. et al. A cross-platform toolkit for mass spectrometry and proteomics. Nature Biotechnology 30, 918-920 (2012).

[24] Consortium, T. U. UniProt: the universal protein knowledgebase in 2021. Nucleic acids research 49, D480-D489 (2021).

[25] Kong, A. T., Leprevost, F. V., Avtonomov, D. M., Mellacheruvu, D. \& Nesvizhskii, A. I. MSFragger: Ultrafast and comprehensive peptide identification in mass spectrometry-based proteomics. Nature Methods 14, 513-520 (2017).

[26] Craig, R. \& Beavis, R. C. TANDEM: Matching proteins with tandem mass spectra. Bioinformatics 20, 1466-1467 (2004).

[27] Dorfer, V. et al. MS Amanda, a universal identification algorithm optimized for high accuracy tandem mass spectra. Journal of Proteome Research 13, 3679-3684 (2014).

[28] Kim, S. \& Pevzner, P. A. MS-GF+ makes progress towards a universal database search tool for proteomics. Nature Communications 5 (2014).

[29] Blumberg, L. M. Theory of gradient elution liquid chromatography with linear solvent strength: Part 2. peak width formation. Chromatographia 77, 189-197 (2014).

[30] Senko, M. W., Beu, S. C. \& Mclafferty, F. W. Determination of Monoisotopic Masses and Ion Populations for Large Biomolecules from Resolved Isotopic Distributions. Journal of the American Society for Mass Spectrometry (1995). 
[31] Suits, F., Lepre, J., Du, P., Bischoff, R. \& Horvatovich, P. Two-dimensional method for time aligning liquid chromatography-mass spectrometry data. Analytical Chemistry 80, 3095-3104 (2008). URL http://pubs .acs .org/doi/abs/10.1021/ ac702267h.

[32] Nesvizhskii, A. I. \& Aebersold, R. Interpretation of Shotgun Proteomic Data: The Protein Inference Problem. Molecular \& Cellular Proteomics 1419-1440 (2005).

[33] Cox, J. et al. Accurate proteome-wide label-free quantification by delayed normalization and maximal peptide ratio extraction, termed MaxLFQ. Molecular \& cellular proteomics : MCP 13, 2513-26 (2014). URL http://www.ncbi.nlm.nih.gov/pubmed/24942700http://www.pubmedcentral . nih.gov/articlerender.fcgi?artid=PMC4159666.

[34] Veselkov, K. A. et al. Optimized Preprocessing of Ultra-Performance Liquid Chromatography/Mass Spectrometry Urinary Metabolic Profiles for Improved Information Recovery Kirill. Analytical Chemistry 83, 5864-5872 (2011).

[35] Benjamini, Y. \& Hochberg, Y. Controlling the false discovery rate: a practical and powerful approach to multiple testing. Journal of the Royal statistical society: series B (Methodological) 57, 289-300 (1995).

[36] Fang, L. et al. Serum and follicular fluid fetuin-B levels are correlated with fertilization rates in conventional IVF cycles. Journal of Assisted Reproduction and Genetics 36, 1101-1107 (2019).

[37] Uhlén, M. et al. Tissue-based map of the human proteome. Science 347 (2015).

[38] Uhlen, M. et al. A pathology atlas of the human cancer transcriptome. Science 357 (2017).

[39] Thul, P. J. et al. A subcellular map of the human proteome. Science 356 (2017).

[40] Gillet, L. C. et al. Targeted Data Extraction of the MS/MS Spectra Generated by Data-independent Acquisition: A New Concept for Consistent and Accurate Proteome Analysis. 2 11, O111.016717 (2012). URL http: //www . mcponline .org/ lookup/doi/10.1074/mcp.0111.016717.

[41] Krishnan, S. et al. Instrument and process independent binning and baseline correction methods for liquid chromatography-high resolution-mass spectrometry 
${ }_{611}$ deconvolution. Analytica Chimica Acta 740, 12-19 (2012). URL http://dx.doi. $612 \quad$ org $/ 10.1016 /$ j.aca.2012.06.014.

613

[42] Caruana, R. A., Searle, R. B., Heller, T. \& Shupack, S. I. Fast Algorithm for the Resolution of Spectra. Analytical Chemistry 58, 1162-1167 (1986).

[43] Guo, H. A simple algorithm for fitting a gaussian function. IEEE Signal Processing Magazine 28, 134-137 (2011).

[44] Leptos, K. C., Sarracino, D. A., Jaffe, J. D., Krastins, B. \& Church, G. M. MapQuant: Open-source software for large-scale protein quantification. Proteomics 6, 17701782 (2006).

[45] Ahmad, I. et al. A high-throughput processing service for retention time alignment of complex proteomics and metabolomics LC-MS data. Bioinformatics 27, 11761178 (2011). 


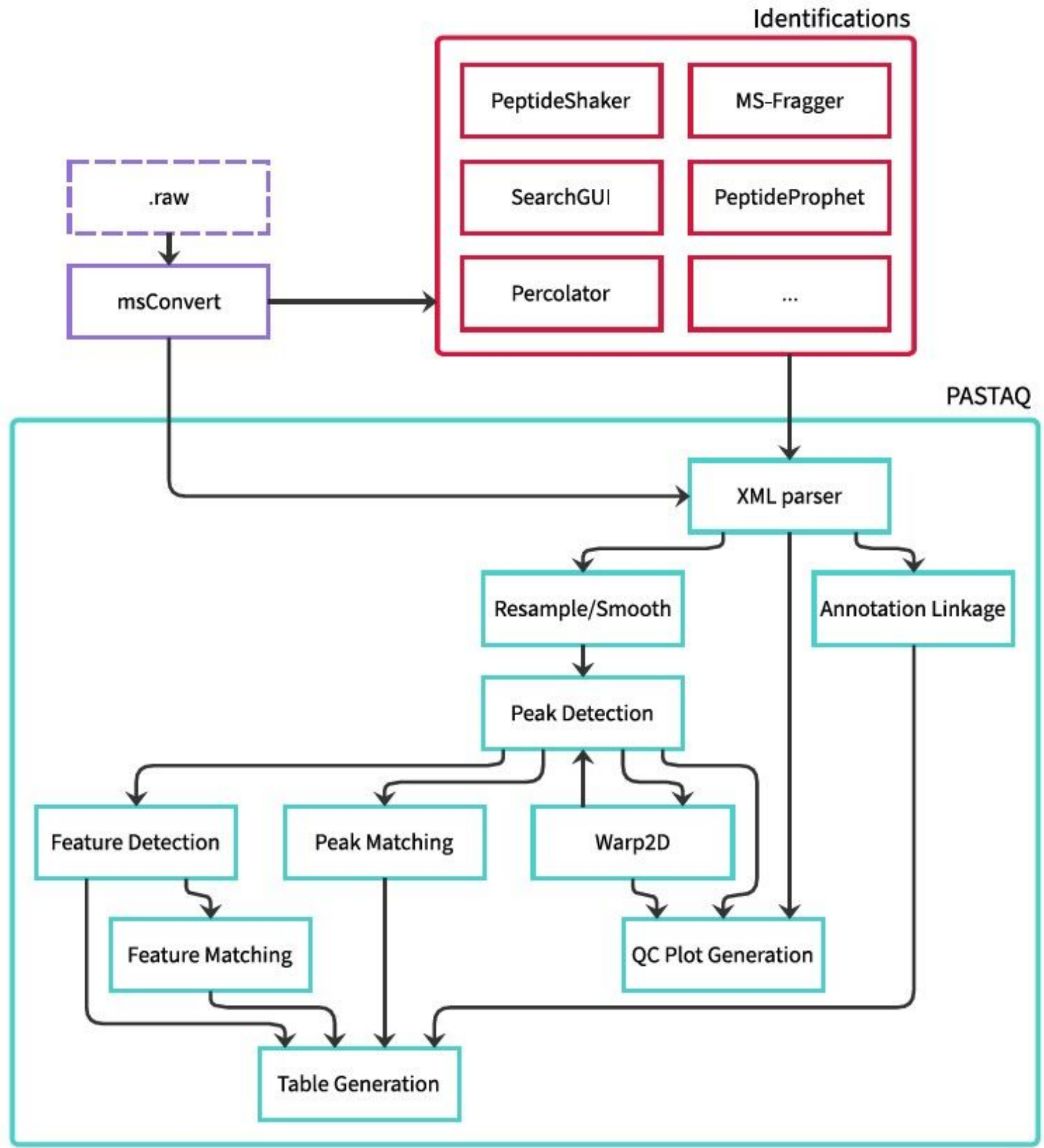

Figure 1

Diagram of the interactions between the main modules in PASTAQ's DDA pipeline. A more detailed diagram can be found in the Supplementary Information (Fig. S7). Note that identifications are not 
required for peak or feature detection by PASTAQ but necessary to link features to identified peptides and ultimately to proteins.

a
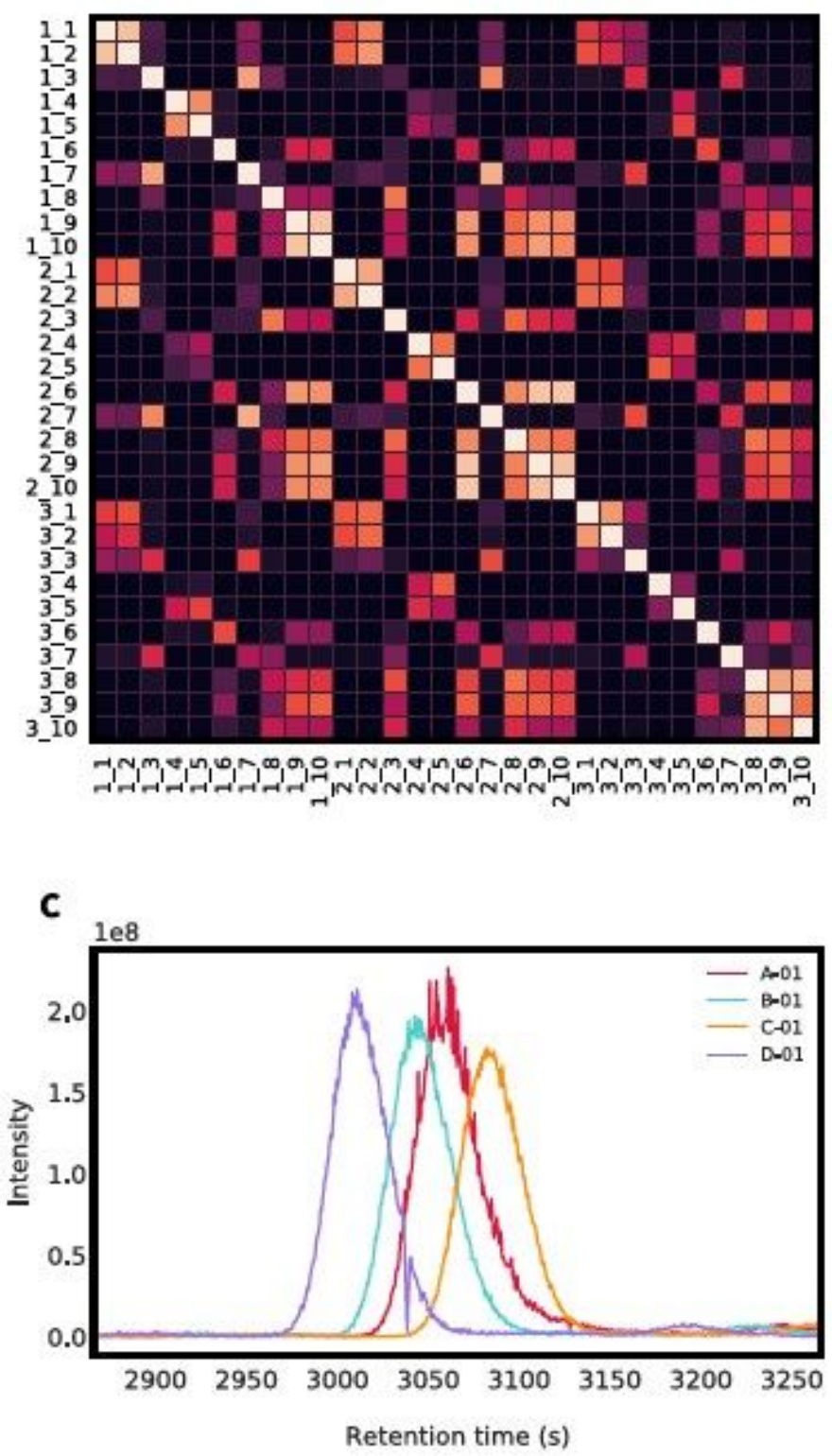

b

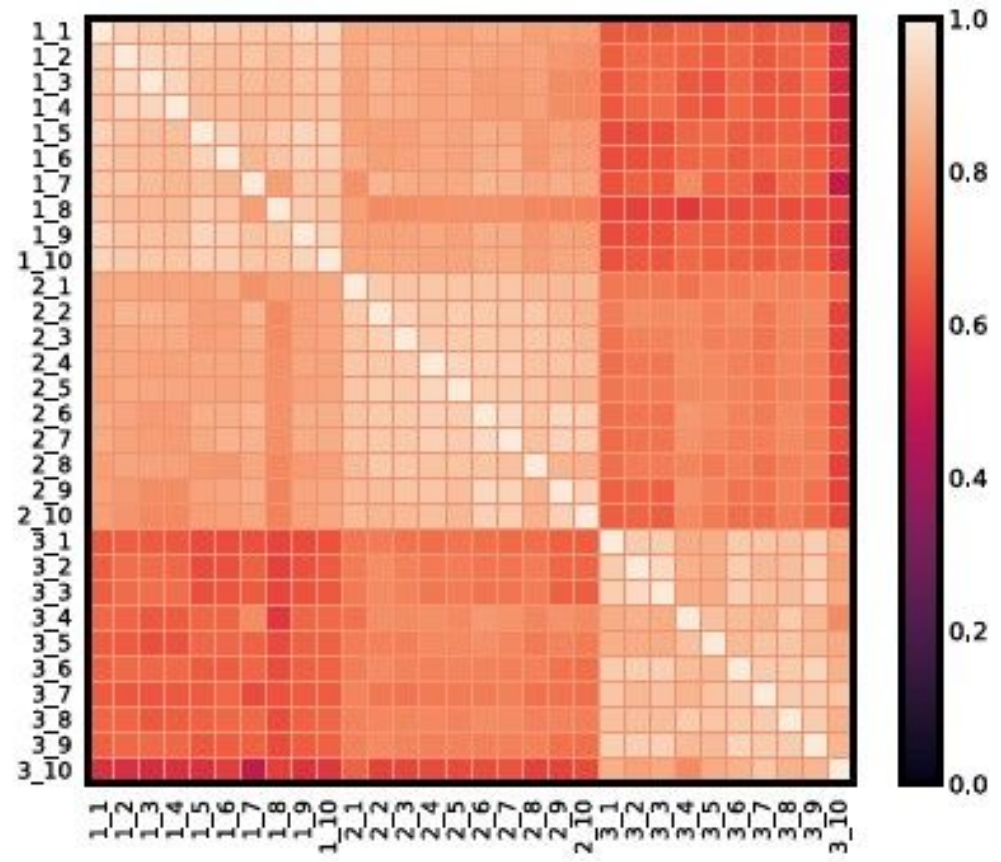

d

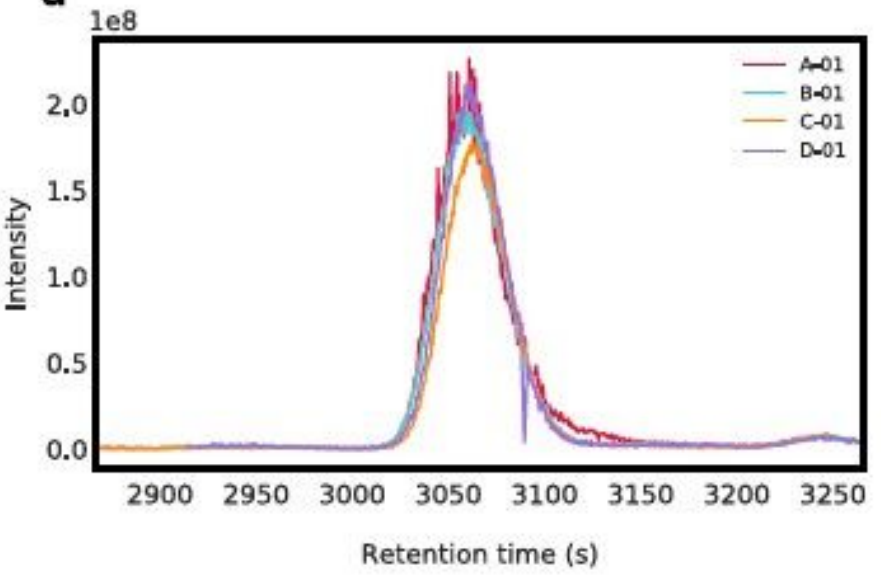

Figure 2

Effect of the retention time alignment on the similarity matrix of the HYE dataset (top) and the extracted ion chromatogram of an isotopic peak from the QconCAT dataset (bottom), before (a, c) and after (b, d) alignment. 

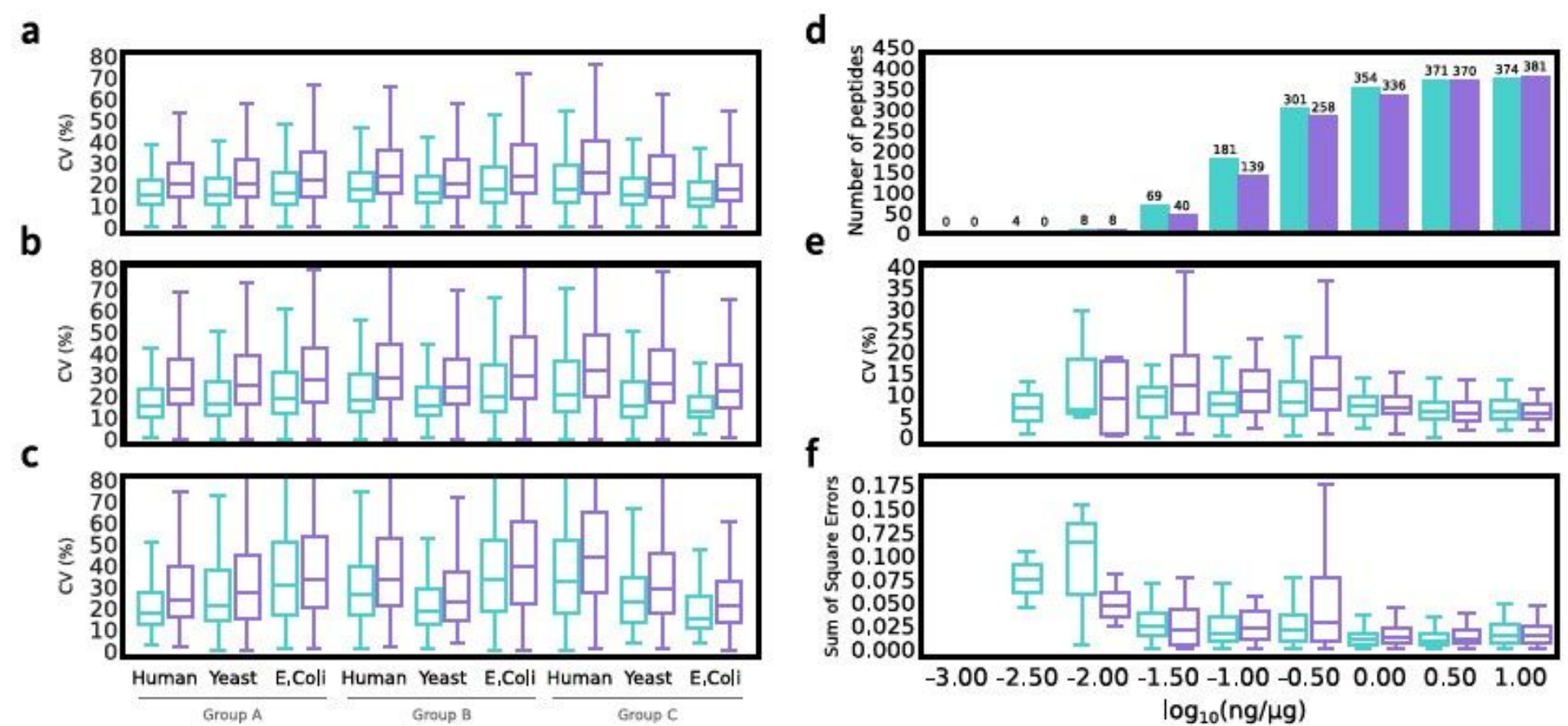

-PASTAQ =- MaxQuant

\section{Figure 3}

(a-c) Comparative of the CV (\%) between PASTAQ quantification with PeptideShaker's consensus identifications, and MaxQuant for (a) features, (b) peptides and (c) proteins in the HYE dataset. (d-f) Evaluation of (d) number, (e) CV and (f) sum of square errors of quantified peptide for each log10 spikedin level in the QconCAT dataset. 

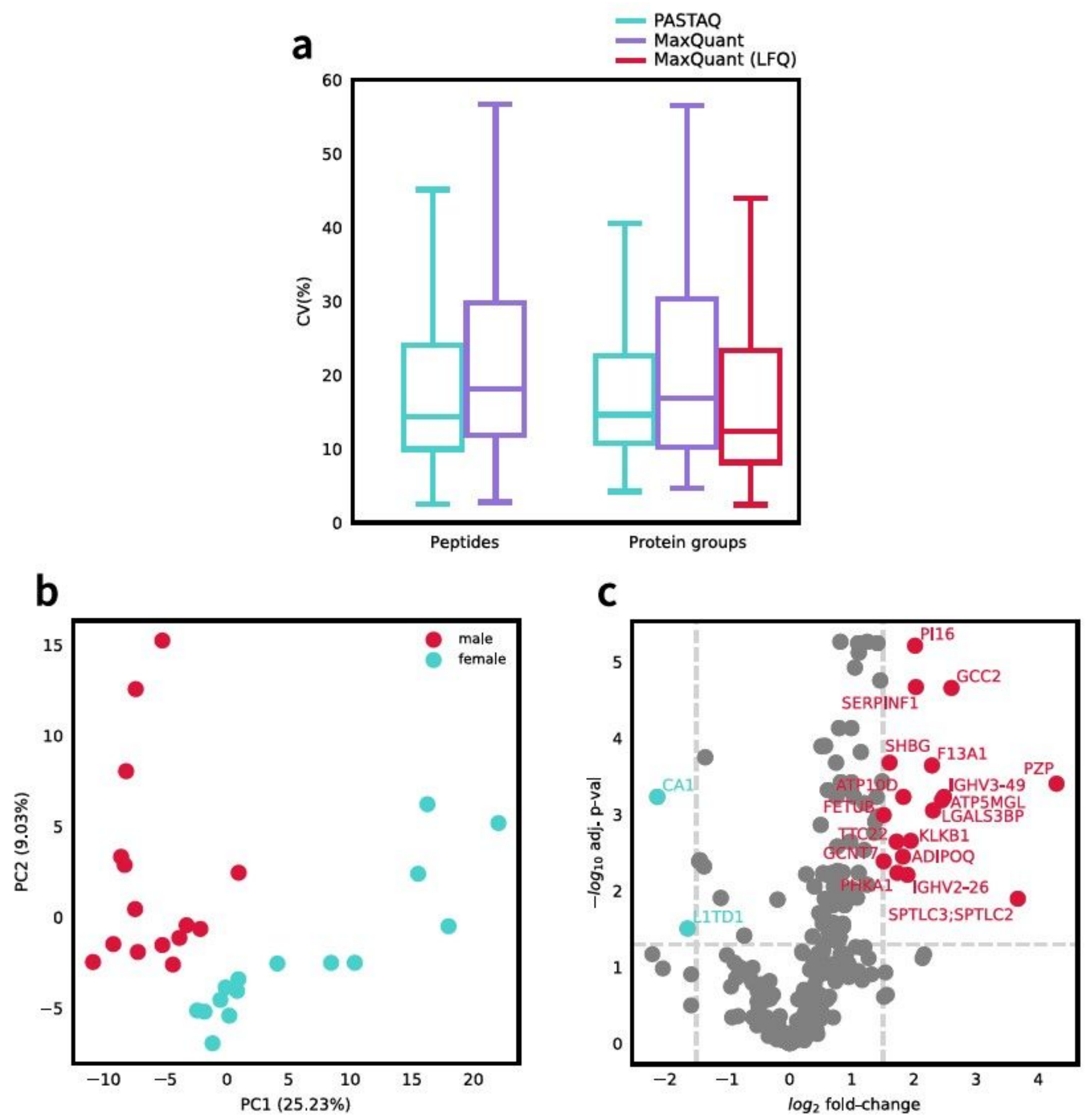

Figure 4

Evaluation of PASTAQ performance on human serum datasets: (a) Distribution of CVs on the peptide and protein group quantification levels for the fifteen technical replicates dataset. (b) Principal Component Analysis (PCA) of the male-female dataset, showing a clear separation between groups. (c) Volcano plot showing selected gender related proteins for the male-female dataset based on the adjusted p-values from Welch's t-test and the log2 fold-change. 


\section{Supplementary Files}

This is a list of supplementary files associated with this preprint. Click to download.

- 20210504pastaqanalchemsupplementary.pdf 